\title{
Effects of reversible lane implementation, a case study simulation
}

\author{
Lucian Lobonț ${ }^{1, *}$, and Andrei Marius Pînzariu ${ }^{2}$ \\ ${ }^{1}$ Lucian Blaga University of Sibiu, Faculty of Engineering, 10 Victoriei Blv. 550024, Sibiu, Romania \\ ${ }^{2}$ S.C. Continental Automotive S.R.L. Salzburg Street, No. 8, Sibiu, Romania
}

\begin{abstract}
In a modern society, transport is a necessity for carrying out certain activities that human communities are involved in. This need for mobility is on a continuous rise and can be characterized by the possibility of moving from one point to another, using different means of transport, in order to facilitate the performed activities. The purpose of this research is to design and evaluate the implementation of a system of reversible lanes using simulation. As methodology, it starts from an analysis of data collected from real traffic condition through implementation and simulation with the Synchro application. The analysis proposes solutions to decongest traffic through the implementation of reversible lanes and the simulation is performed again for impact assessment. Our results suggest that the implementation of reversible lanes can help to decongest traffic if complementary solutions are adopted. The approach can be used successfully in any scientific analysis and design approach to optimize traffic flows.
\end{abstract}

\section{Introduction}

In a modern society transport is a necessity for carrying out certain activities that human communities carry out. This need for mobility is on a continuous rise and can be characterized by the possibility of moving from one point to another, using different means of transport, in order to facilitate the performed activities.

In urban communities, transport is carried out in different ways such as roads, overhead and underground railways, water or air, for the purpose of passenger transport or freight transport. The movement must be done in the shortest possible time, safely [1] and at minimal cost.

The development of cities and the increase in the number of inhabitants in the urban area have led to the increase in the number of means of transport which contradicted the road infrastructure, which was designed and adapted for the most part for reduced traffic.

Due to the centralized nature of urban traffic congestion takes shape in the morning from the residential areas to the industrial areas and in the opposite direction in the afternoon. The need to find solutions for the decongestion of urban road traffic is continuous. Different methodologies for traffic optimization are used, such as:

\footnotetext{
* Corresponding author: lucian.lobont@ulbsibiu.ro
} 
mathematical models [2], statistical tools [3], microscopic and mesoscopic modeling [4-5], traffic simulation [6].

The reversible lanes are the lanes were traffic can go in either direction, depending on certain conditions. Implementation of reversible lanes can be found on tunnels and bridges but also in other places were the objective was the limitation of the effects of congestion and for traffic optimization. The reversible lane solution is not a novelty and according to Wolshon and Lambert [7] this solution is applied from over 70 years all over the world, especially in United States of America. Researches in this area were also performed [8]. However, in Europe the system of reversible lanes is not a common approach.

The purpose of the research is to design and evaluate the implementation of a system of reversible lanes using simulation in order to reduce traffic congestion.

\section{Methods}

The steps used in performing our research were the following:

- Establishing the road artery used for analysis;

- Collecting data from the selected road;

- Computing the data regarding the specific flow traffic indicators;

- $\quad$ Preparing the simulation model;

- Implementing the proposed solution;

- Evaluating the results.

For the simulation we used Synchro application which is a macroscopic analysis and optimization software. (https://www.trafficware.com/synchro.html)

\section{Results}

\subsection{Establishing the road artery used for analysis}

The analysis was carried out in the city of Sibiu. The city of Sibiu has an area of $121 \mathrm{~km}^{2}$, with a population of 147245 inhabitants, with a density of 1273 places $/ \mathrm{km}^{2}$. It consists of 21 districts, with more than 650 nominated streets, totaling more than 150 kilometers in length. The local public transport is carried out by the transport company Tursib, which manages the routes of public transport within the municipality.

The traffic inside Sibiu is composed of almost all groups of vehicles and pedestrians, trams and trolleybuses missing from the traffic system. The infrastructure has undergone major changes over time, with main and secondary streets being upgraded and adapted to current traffic, and many of the intersections become roundabout intersections, reaching a number close to 40 .

The road section we selected for our analysis is one of the main transit arteries going to one of the most industrialized area in western Sibiu. The selected road section is represented in figure 1 by the marked oval. A detailed view is depicted in figure 2. The type of the intersection from the analyzed area is roundabout. 


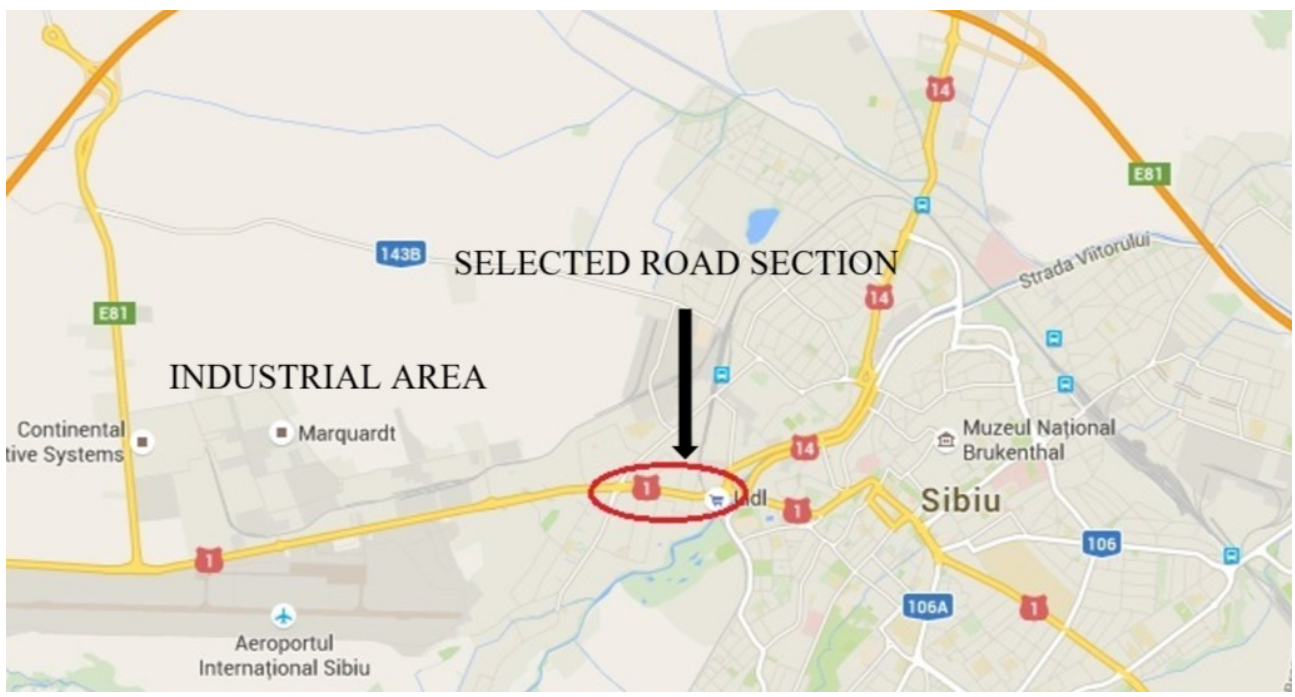

Fig. 1. Selected road artery. (caption from https://www.google.ro/maps/)

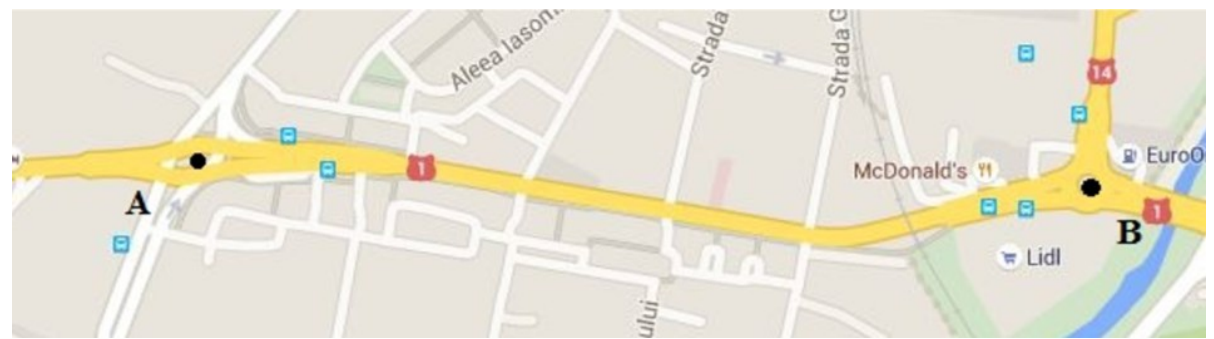

Fig. 2. Detailed view of selected artery. (caption from https://www.google.ro/maps/)

The composition and dimensional characteristics of the road are presented in figure 3 . The road is a two-way road with two lanes on each way.

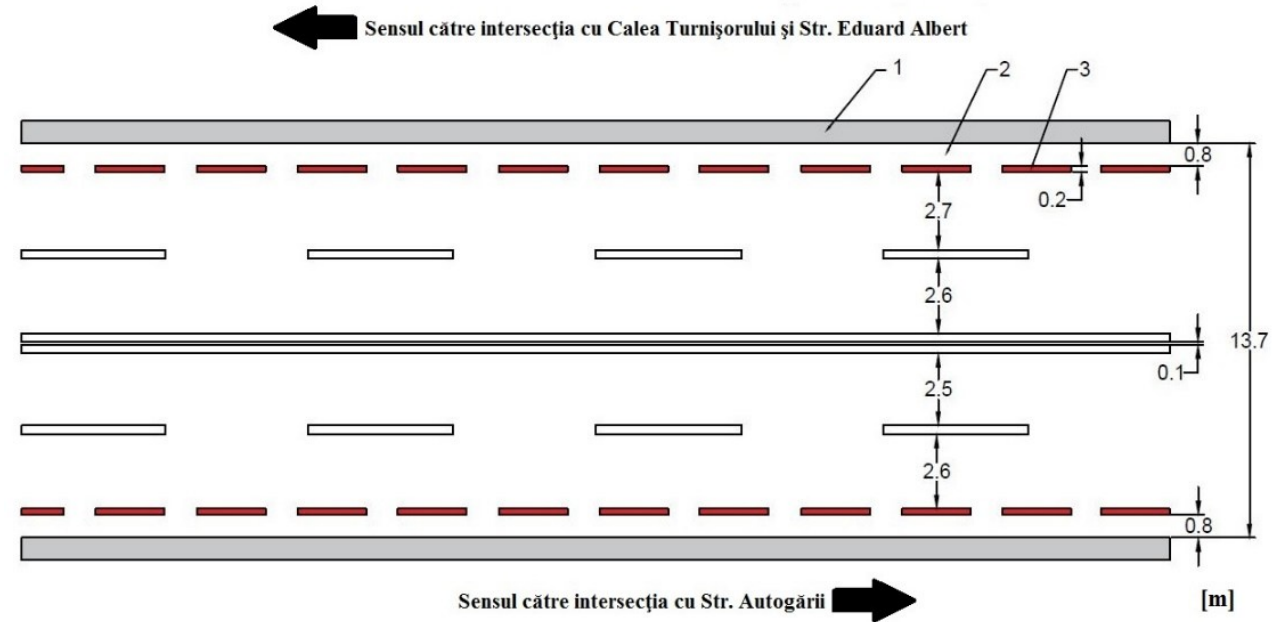

Fig. 3. Dimensional characteristics of the road. 


\subsection{Data collection}

From our previous observation the flow of traffic is congested during the hours 16:30 18:30, when the employees from the factories in the industrial area are coming back to the city. The direction is from outside the city to the city. The data were collected from the two main intersections marked with $\mathrm{A}$ and $\mathrm{B}$ in the figure 2 . The traffic congestion can be clearly seen in figure 4.

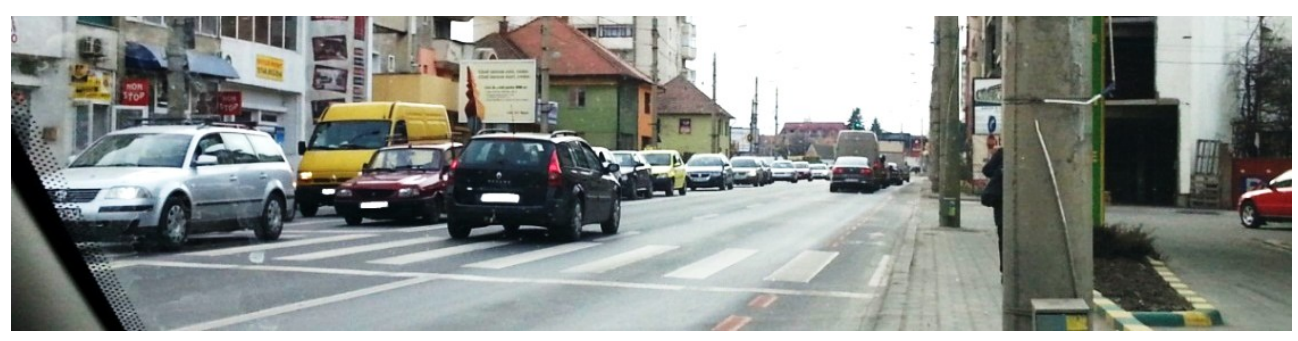

Fig. 4. Traffic congestion on analyzed road artery.

The method used for collecting data was video capturing of the traffic flow in the selected intersections and then counting the vehicles from the video file.

The following data were collected: the number of vehicles entering the intersection from each arm and the type of vehicles entering the intersection. The collecting data were split in fifteen minutes time slots and then the total number of vehicles counted were converted from physical vehicles to passenger car equivalent (PCE) according to standard SR 7348:2001 - Road works. Vehicles equivalent for traffic capacity determination. The data were collected during five week days from Monday to Friday in order to cover the busiest time of the day. After the data collection we have calculated the mean number of vehicles expressed as passenger car equivalent.

The results from intersection A are presented in the table 1.

Table 1. Data collection from A intersection

\begin{tabular}{|c|c|c|c|c|c|c|c|c|c|c|c|c|}
\hline \multirow[b]{2}{*}{ Mean } & \multicolumn{3}{|c|}{ Airport - Center } & \multicolumn{3}{|c|}{ Center - Airport } & \multicolumn{3}{|c|}{ Cl. Turnişorului Str. } & \multicolumn{3}{|c|}{ Ed. Albert Str. } \\
\hline & Left & Forward & Right & Left & Forward & Right & Left & Forward & Right & Left & Forward & Right \\
\hline Passenger car & 64 & 292 & 19 & 51 & 163 & 39 & 41 & 21 & 29 & 13 & 22 & 51 \\
\hline Mini buses & 6 & 22 & 1 & 7 & 13 & 2 & 5 & 2 & 3 & 2 & 3 & 2 \\
\hline Large Buses & 0 & 20 & 0 & 2 & 11 & 0 & 0 & 0 & 0 & 0 & 0 & 2 \\
\hline Trucks & 2 & 18 & 0 & 0 & 13 & 0 & 0 & 0 & 2 & 1 & 0 & 0 \\
\hline Bicycle, Motorcycle & 0 & 1 & 0 & 0 & 0 & 0 & 0 & 0 & 0 & 0 & 0 & 0 \\
\hline Total & 72 & 353 & 20 & 60 & 200 & 41 & 46 & 23 & 34 & 16 & 25 & 55 \\
\hline $\begin{array}{c}\text { Total } \\
\text { vehicle/intersection } \\
\text { arm }\end{array}$ & & 445 & & & 301 & & & 103 & & & 96 & \\
\hline
\end{tabular}

The results from intersection B are presented in table 2. 
Table 2. Data collection from B intersection

\begin{tabular}{|c|c|c|c|c|c|c|c|c|c|}
\hline \multirow[b]{2}{*}{ Mean } & \multicolumn{3}{|c|}{ Airport - Center } & \multicolumn{3}{|c|}{ Center - Airport } & \multicolumn{3}{|c|}{ Autogării Str. } \\
\hline & Left & Forward & Right & Left & Forward & Right & Left & Forward & Right \\
\hline Passenger car & 12 & 338 & 7 & 11 & 229 & 33 & 106 & 5 & 90 \\
\hline Mini buses & 2 & 17 & 0 & 2 & 19 & 4 & 9 & 0 & 8 \\
\hline Large Buses & 1 & 19 & 0 & 0 & 9 & 0 & 10 & 0 & 9 \\
\hline Trucks & 2 & 33 & 0 & 0 & 12 & 2 & 2 & 0 & 5 \\
\hline Bicycle, Motorcycle & 0 & 2 & 0 & 0 & 1 & 0 & 0 & 0 & 0 \\
\hline Total & 17 & 409 & 7 & 13 & 270 & 39 & 127 & 5 & 112 \\
\hline $\begin{array}{c}\text { Total } \\
\text { vehicle/intersection } \\
\text { arm }\end{array}$ & & 433 & & & 322 & & & 244 & \\
\hline
\end{tabular}

\subsection{Computing the data}

For each intersection we calculated the traffic stream parameters. Due to the limitations of the paper length we will present only the most relevant of them and the final results.

For intersections A and B we have determined: traffic intensity, traffic density, traffic speed, intersection capacity and level of service (LOS). The traffic speed for entering the intersection A resulting from our calculations is presented in figure 5 and the traffic capacity of A intersection is 5401 vehicles/hour. The level of service was determined according to table 3 and for $\mathrm{A}$ intersection is $\mathrm{F}$.

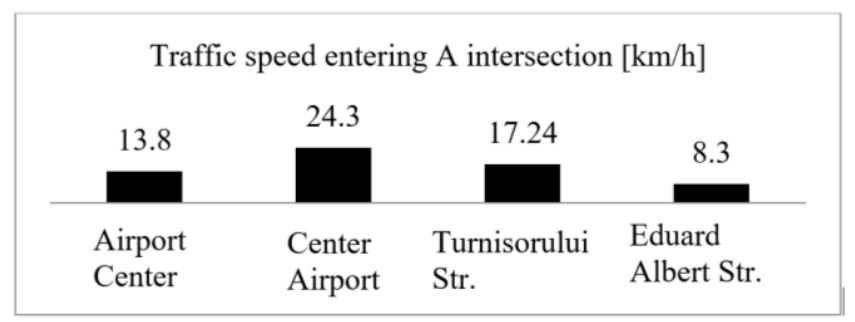

Fig. 5. Traffic speed for entering in intersection A.

Table 3. Level of service for roundabout intersection

\begin{tabular}{|c|c|}
\hline Level of service & Delay [s/veh] \\
\hline A & $<10$ \\
\hline B & $10-15$ \\
\hline C & $15-25$ \\
\hline D & $25-35$ \\
\hline E & $35-50$ \\
\hline F & $>50$ \\
\hline
\end{tabular}

The traffic speed for entering the intersection B resulted from our calculations is presented in figure 6 and the traffic capacity of B intersection is 5640 vehicles/hour. The level of service was determined according to table 3 and for B intersection is F. 


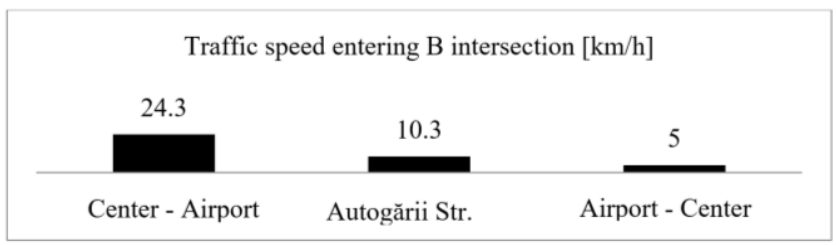

Fig. 6. Traffic speed for entering in intersection B.

\subsection{Preparing the simulation model}

The simulation model was established using Synchro application. This software application allows to create the road model respecting all the constructive characteristics such as lanes, intersections, geometrical dimensions. For a realistic simulation all the traffic parameters can be inserted. After simulation the application can offer different traffic parameters, various reports and a graphical view of the traffic.

As can be seen in figure 7 the simulation model was prepared and the traffic volumes for each arm of the intersections was established (fig. 8).

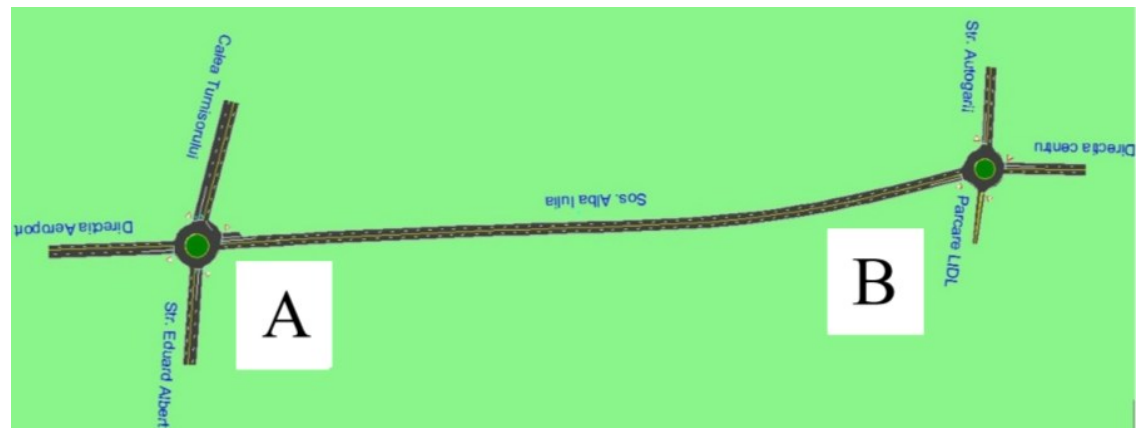

Fig. 7. Simulation model
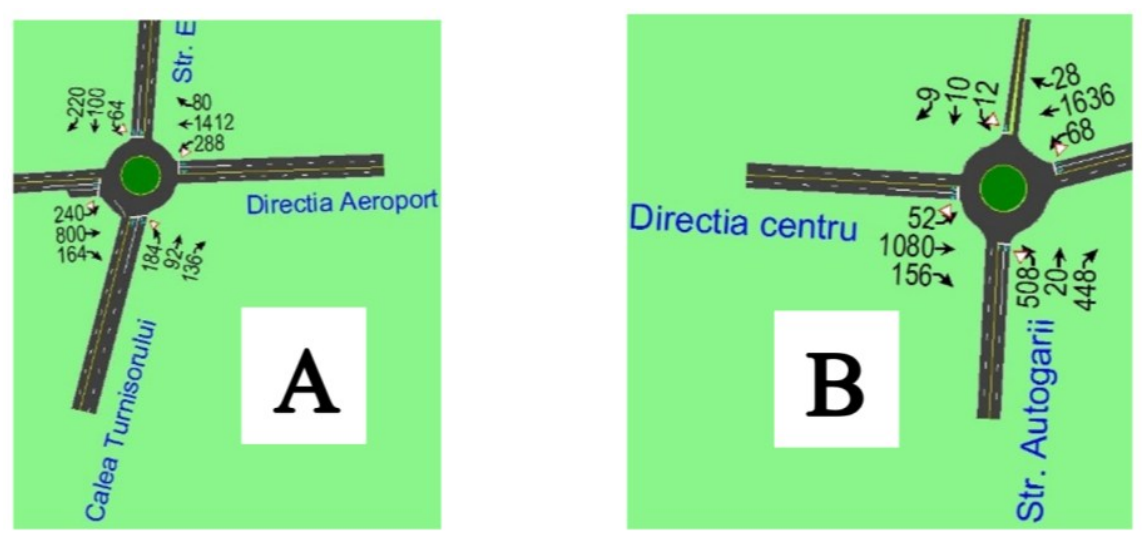

Fig. 8. Traffic volumes

The results of the initial simulation (fig. 9) confirm the situation from the reality and indicate that the flow of traffic is congested from intersection A to intersection B. These results are also a validation of our model. 


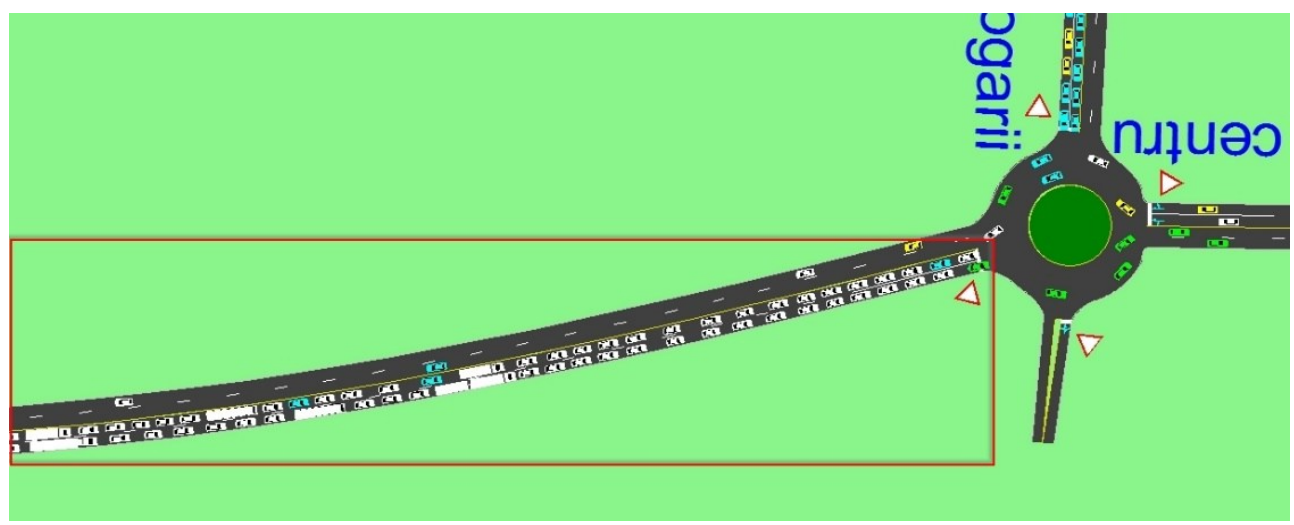

Fig. 9. Simulation of the real traffic situation and validation of the model

\subsection{Implementing the proposed solution}

The solution proposed was to create a third lane on the congested direction and to limit the opposite direction to one lane using the reversible lane approach. The simulation results are presented in figure 10 .

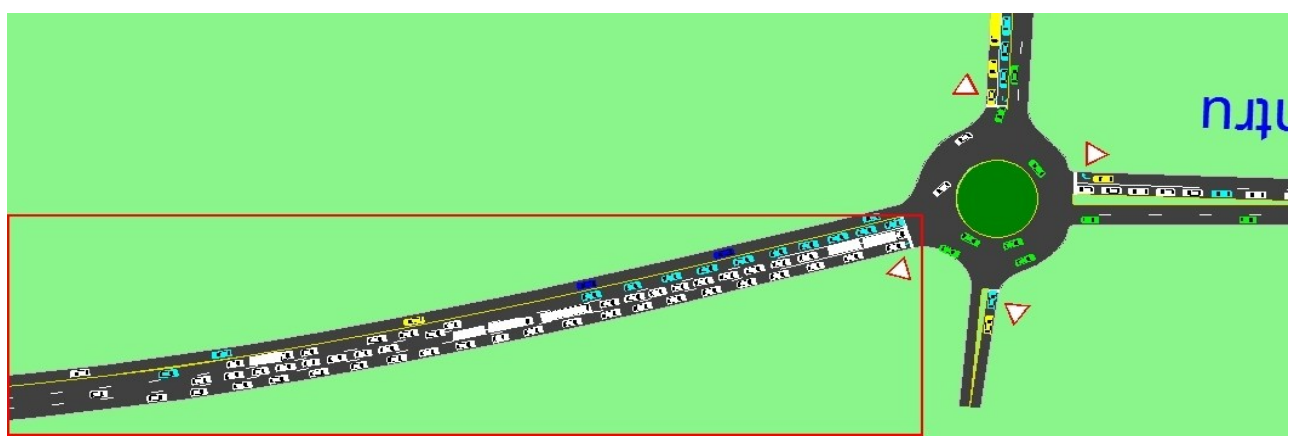

Fig. 10. Simulation of the proposed solution

\section{Discussion}

Our analysis was focused on identifying the changes in traffic flow following the implementation of a reversible lane solution on a road artery on Sibiu county. The values of intersection capacity utilization (ICU) obtained from the simulation are presented in figure 11 - before reversible line implementation and figure 12 - after reversible lane implementation. The ICU can be used to foresee how frequently an intersection will experience congestion and is related with the level of service (LOS). Values of ICU over $100 \%$ suggest that the analyzed intersection is over capacity and experiences congestion periods over 120 minutes/day. Analyzing the values resulted from the simulation, we obtained an improved value in the intersection $\mathrm{B}$, but worse values in intersection $\mathrm{A}$. The results are not satisfactory and in the first phase we are tempted to reject this solution. We consider that further analysis should be done on reconfiguring the geometry of the intersections in order to reduce the ICU and to transform the reversible lane solution into a viable solution. 


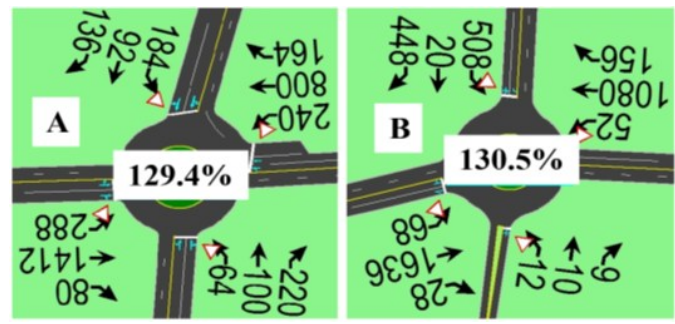

Fig. 11. ICU values before implementing the solution.

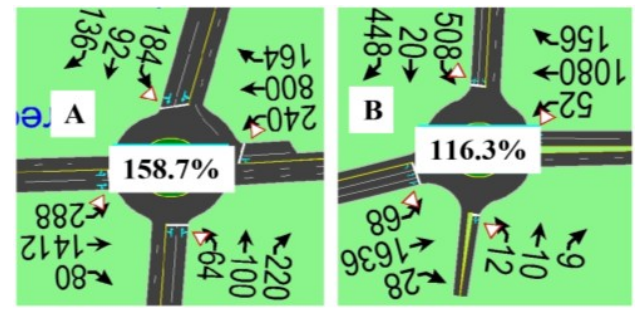

Fig. 12. ICU values after implementing the reversible lane solution.

\section{Conclusions}

Urban traffic has developed as urban communities began to evolve, and the needs to transport and move began to grow. It influenced and in turn was influenced by the characteristics of economic and social development. Urban transport issues are boosted by population growth, the growth of production and other activities, and the increase in the number of vehicles. Also, there is no need to neglect the flow of vehicles that in the morning is congested to the factories and workplaces and in the afternoon traffic values increases to the city.

Finding new solutions for optimizing and optimizing road traffic flows is a major priority from the point of view of the development of society, increasing the standard of living and the level of industrialization. Solutions can go from paths already traveled, but mostly from innovative approaches. For decision-makers, the use of modeling and simulation tools is vital. These tools can provide major benefits such as reducing the time needed to implement projects, detailed analyzes, applying and evaluating various scenarios, reducing costs. The approach used in this research can be successfully applied in any scientific analysis and design approach to optimize traffic flows.

\section{References}

1. L. Lobont, A.C. Filichi, L.G. Popescu, AUO-FMTE, 1, pp. 175-180 (2013)

2. S. Salman, S. Alaswad, Comput. Oper. Res. 99, pp.191-205 (2018)

3. Z. Tiana, L. Jiaa, H. Donga,F. Sua, Z. Zhangc, Procedia Eng. 137, pp.537-546, (2016)

4. M. Zlatkovica, S. Zlatkovicb, T. Sullivanc, J. Bjornstadd, S. K. F. Shahandashtie, Sustain Cities Soc, 46, pp.101409 (2019)

5. J. Brugmann, M. Schreckenberg, W. Luther, Simul Model Pract Theory, 48, pp.58-92 (2014)

6. J.Jin,X. Ma, I.Kosonen, Adv Eng Softw, 114, pp.348-360 (2017)

7. B. Wolshon, L. Lambert, J Transp Eng, 12, 132 (2006)

8. J. Wu, H. Sun, Z.Y. Gao, H.Z. Zhang, Eng Optimiz. 41, pp.87-97 (2009) 